\title{
El empoderamiento del alumno: una tendencia favorable en la educación superior
}

Student empowerment a favorable trend in higher education Empoderamento do aluno: uma tendência favorável no ensino superior

\author{
Manuel Higinio Morales García \\ Universidad Juárez Autónoma de Tabasco, México \\ moralesgarciamh@hotmail.com \\ https://orcid.org/0000-0003-2560-6300 \\ Carlos Alberto Balcázar Nájera \\ Universidad Juárez Autónoma de Tabasco, México \\ Kail_11@hotmail.com \\ https://orcid.org/0000-0002-6228-7903 \\ Heberto Romeo Priego Álvarez \\ Universidad Juárez Autónoma de Tabasco, México \\ heberto_priego@hotmail.com \\ https://orcid.org/0000-0001-9217-5702 \\ Jonatan Flores Morales \\ Universidad Juárez Autónoma de Tabasco, México \\ jonatanfm@hotmail.com \\ https://orcid.org/0000-0002-6567-7237
}

Autor por correspondencia: Manuel Higinio Morales García, moralesgarciamh@hotmail.com 


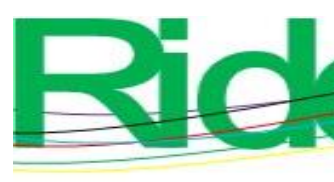

Revista Iberoamericana para la Investigación y el Desarrollo Educativo ISSN $2007-7467$

\section{Resumen}

Las nuevas teorías de los procesos de enseñanza en todos los niveles se han separado de las pasadas metodologías del aprendizaje en donde en algunas se priorizaba más la transmisión del conocimiento; sin embargo, actualmente esta tendencia ha cambiado, pues el alumno ahora es concebido como el principal actor en el proceso de enseñanza-aprendizaje. Objetivo: Se determinó el empoderamiento del estudiante universitario ante las nuevas prácticas educativas en la educación superior. Métodos: Se realizó una investigación exploratoria, descriptiva y transversal; la muestra estuvo constituida por 113 alumnos a los cuales se aplicó un instrumento tipo encuesta conformado por cuatro apartados correspondiente a las siguientes variables: desarrollo de contenidos temáticos, herramientas didácticas, apoyo docente e interacción del alumno y profesor. Resultados: Se destaca la percepción de los alumnos en cuanto al desarrollo de contenidos temáticos por parte del profesor; en concreto, $43 \%$ consideró una mayor inclinación hacia los contenidos prácticos y $36.28 \%$ hacia los contenidos teóricos; esto se suele realizar mediante el uso de herramientas didácticas tales como las maquetas, el pizarrón y las plataformas virtuales. Asimismo, resultaron relevantes los factores que intervienen en la interacción profesoralumno, tales como la puntualidad, el conocimiento y la práctica ética. Conclusiones: Se muestra la participación del estudiante relacionado con las expectativas en su formación profesional, enfatizando mejoras en los contenidos curriculares, en las actividades extracurriculares de los programas de estudios, así como una docencia centrada en el acompañamiento y dirección del estudiante.

Palabras claves: capacitación, docencia, enseñanza profesional, gestión educativa, liderazgo.

\section{Abstract}

The new theories of teaching processes at all levels have been separated from past learning methodologies where in some the transmission of knowledge was given more priority, currently this trend has changed focusing the student as the main actor in the teachinglearning process . Objective: the empowerment of the university student in the face of new educational practices in higher education was determined. Methods: An exploratory, descriptive and cross-sectional research was carried out, the sample consisted of 113 students, a survey-type instrument was applied, consisting of four sections corresponding to 


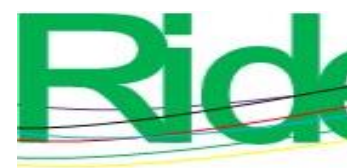

Revista Iberoamericana para la Investigación y el Desarrollo Educativo ISSN $2007-7467$

the study variables; development of thematic content, didactic tools, teaching support and interaction of the student and teacher Results: The perception of the students regarding the development of thematic content on the part of the teacher stands out, $43 \%$ considered a greater inclination towards practical content and $36.28 \%$ towards the theoretical contents, accentuated by a frequent use of didactic tools such as models, pintarrón and virtual platforms, respectively, the factors that intervene in the teacher-student interaction such as punctuality, knowledge and ethical practice are relevant. Conclusions: The participation of the student is shown related to the expectations in their professional training, emphasizing improvements in the curricular contents, in the extracurricular activities of the study programs, as well as a teaching focused on the accompaniment and direction of the student.

Keywords: training, teaching, professional teaching, educational management, leadership

\section{Resumo}

As novas teorias dos processos de ensino em todos os níveis foram separadas das metodologias de aprendizagem anteriores, onde em algumas a transmissão de conhecimento foi mais priorizada; No entanto, essa tendência agora mudou, pois o aluno passou a ser concebido como o principal ator do processo ensino-aprendizagem. Objetivo: Determinouse o empoderamento do estudante universitário frente às novas práticas educacionais no ensino superior. Métodos: Foi realizada uma investigação exploratória, descritiva e transversal; A amostra foi composta por 113 alunos, aos quais foi aplicado um instrumento do tipo survey, composto por quatro seções correspondentes às seguintes variáveis: desenvolvimento do conteúdo temático, ferramentas didáticas, apoio ao ensino e interação aluno-professor. Resultados: Destaca-se a percepção dos alunos quanto ao desenvolvimento dos conteúdos temáticos pelo professor; especificamente, $43 \%$ consideram uma maior inclinação para os conteúdos práticos e 36,28\% para os conteúdos teóricos; Geralmente, isso é feito por meio do uso de ferramentas didáticas como maquetes, quadro-negro e plataformas virtuais. Da mesma forma, os fatores que interferem na interação professor-aluno, como pontualidade, conhecimento e prática ética, foram relevantes. Conclusões: Mostra-se a participação do aluno relacionada às expectativas em sua formação profissional, enfatizando melhorias nos conteúdos curriculares, nas atividades extracurriculares dos programas de estudos, bem como um ensino voltado para o acompanhamento e direcionamento do aluno.

Palavras-chave: formação, ensino, ensino profissional, gestão educacional, liderança. 


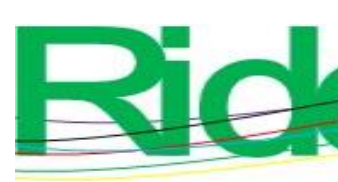

Revista Iberoamericana para la Investigación y el Desarrollo Educativo ISSN 2007-7467

\section{Introducción}

Los procesos de enseñanza de todos los niveles se han separado de las viejas teorías del aprendizaje en las cuales se priorizaba la transmisión del conocimiento. Esto ha generado un cambio de paradigmas cuyos resultados han permitido al alumno fomentar su sentido crítico con el fin de comprender su contexto y transformar su realidad (Blancas, 2018).

En efecto, la nueva práctica docente, en especial la de nivel superior, ahora exige una actualización constate para cumplir con las expectativas de los estudiantes, de ahí que se requieran profesores capaces de desarrollar sus competencias y de trabajar en la mejora de los planes y programas existentes. Para ello, la práctica docente debe centrar sus esfuerzos en tres factores esenciales: los aspectos didácticos (es decir, la planeación y la determinación del ambiente de trabajo con el grupo), el desarrollo pedagógico apegado a los objetivos del programa y la transformación del nuevo conocimiento en el alumno (Moreno Olivos, 2018). Para esto último, sin embargo, se necesita de la participación activa del estudiante, el cual se constituye como el principal protagonista del proceso de enseñanza.

En este sentido, se ha discutido mucho sobre los procesos mediante los cuales el alumno asimila un nuevo conocimiento, entre los que destacan el involucramiento del estudiante en el análisis de las ideas, la adaptación del alumno en sus esquemas mentales para habituarse al pensamiento crítico, abstracto y reflexivo, la corresponsabilidad con el profesor sobre la construcción del propio aprendizaje, así como la participación comprometida en el desarrollo de los contenidos de los programas académicos (Arce-Medina, 2008).

En este contexto de diversas situaciones, unas de las metodologías que ha tenido un impacto positivo en el aprendizaje del alumno es aquella que promueve las actividades cooperativas, las cuales parecieran ofrecer mejores resultados que los modelos individualistas. Esto se podría deber a que con las primeras los estudiantes actúan como protagonistas de sus procesos de aprendizaje y les facilitan las herramientas necesarias para su formación profesional (Guerra Santana, Rodríguez Pulido y Rodríguez, 2019).

Asimismo, se ha percibido que la interacción armónica entre los alumnos y profesores ofrece grandes beneficios, pues la calidad de este intercambio puede aumentar o menoscabar aspectos motivaciones que también juegan un papel esencial en el proceso de aprendizaje (García-Rangel, García Rangel y Reyes Angulo, 2014). Sobre esta variable, la identificación de las características del perfil académico del profesor que requiere el estudiante universitario 


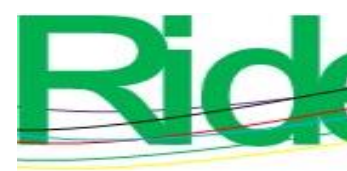
Revista Iberoamericana para la
Investigación y el Desarrollo Educativo
ISSN $2007-7467$

debe ser el objetivo para establecer la capacitación, actualización e incluso la contratación de los profesores universitarios, los cuales deben promover la autonomía del aprendiz. Para esto, no obstante, deben conocer los objetivos, expectativas y necesidades del alumno en su proceso de formación (Liesa, 2018).

El alumno, por tanto, debe ser concebido como un eje sobre el cual gira la práctica docente. Esto significa, en palabras de Zabalza Beraza (2011), que los profesores deben ceder el protagonismo, el discurso y el tiempo a los estudiantes con el fin de generar contextos más participativos e incluyentes. Esto debe suceder porque se ha demostrado que los alumnos aprenden y se motivan más por las actividades cuando se les permite desarrollar su razonamiento y cuando pueden reconstruir y problematizar una situación de su interés (Molina, 2018). Por eso, resulta indispensable conocer cuáles son las principales necesidades académicas de los alumnos para determinar aquellos factores que puedan incidir en su (des)motivación y, en el peor de los casos, en su abandono escolar, realidad que ninguna institución de educación superior debe aceptar.

\section{Método}

La presente fue una investigación de tipo exploratorio, descriptivo y transversal mediante la cual se procuró determinar la participación activa del estudiante como tendencia favorable en la educación superior. La muestra estuvo constituida por 113 alumnos — de un universo de 160 estudiantes - del tercer y cuarto ciclo de la licenciatura en Cirujano Dentista de la Universidad Juárez Autónoma de Tabasco. Para su cálculo se consideró $95 \%$ de confiabilidad y un margen de error de $5 \%$.

Para la recolección de los datos se aplicó un instrumento tipo encuesta conformado por cuatro apartados correspondiente a las variables de estudio: 1) desarrollo de contenidos temáticos, 2) herramientas didácticas, 3) apoyo docente y 4) interacción del alumno-profesor. El instrumento estuvo integrado por 6 preguntas, y fue previamente piloteado y validado mediante el índice de Cronbach, proceso en el cual obtuvo un 0.80 de confiabilidad. La investigación se desarrolló durante los meses de febrero y marzo del año 2020. 


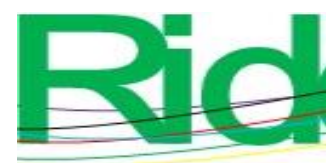

Revista Iberoamericana para la
Investigación y el Desarrollo Educativo
ISSN $2007-7467$

\section{Resultados}

Como se mencionó, fueron encuestados 113 alumnos de manera aleatoria y bajo su consentimiento. A continuación, en la figura 1 se observa que $43 \%$ de los participantes consideraron que el profesor dio prioridad a los contenidos prácticos, $36.28 \%$ a los contenidos teóricos y solo $16.81 \%$ refirió un equilibrio entre esas dos áreas.

Figura 1. Prioridad del profesor en el desarrollo de contenidos temáticos

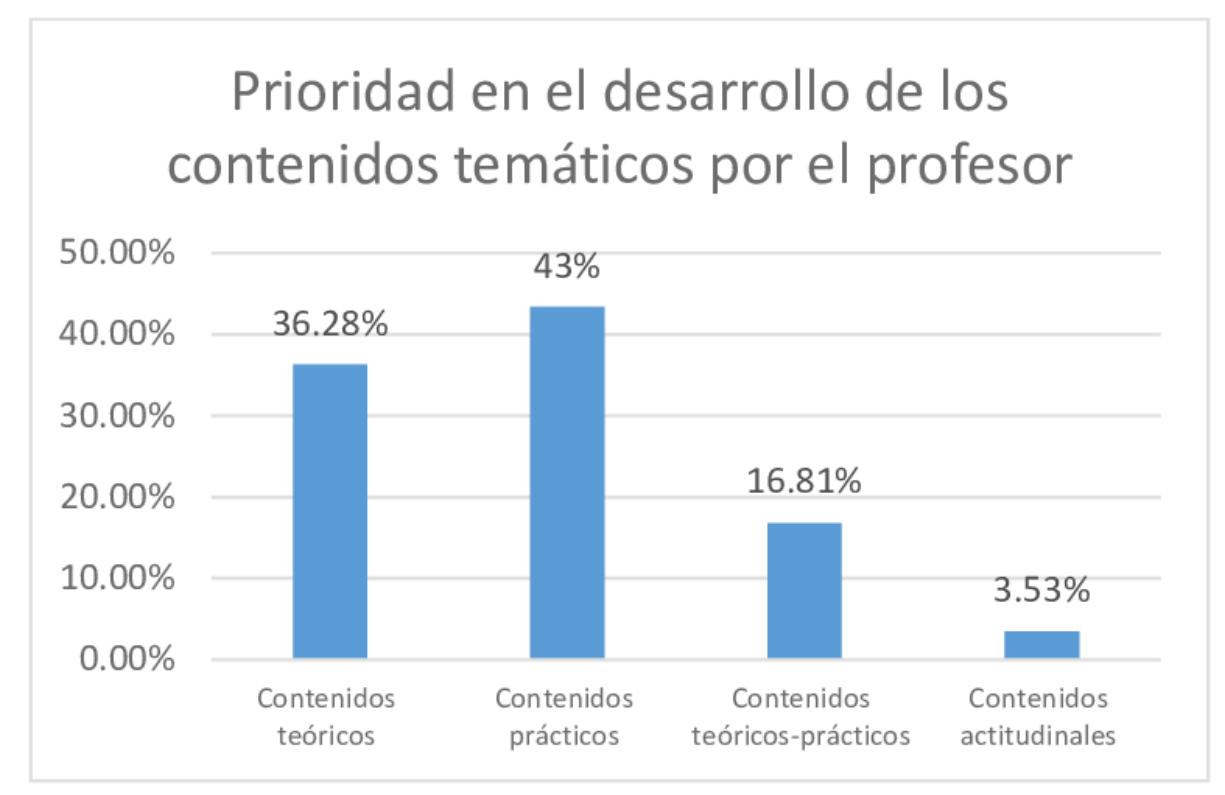

Fuente: Elaboración propia

En relación con la percepción de disfrute de las clases por parte del alumno, $58.40 \%$ indicó que a veces lo hace, mientras que 9.73 \% casi nunca lo hace. Solo $15.04 \%$ expresó que siempre disfruta el desarrollo de las clases. 
Figura 2. El alumno disfruta de las clases

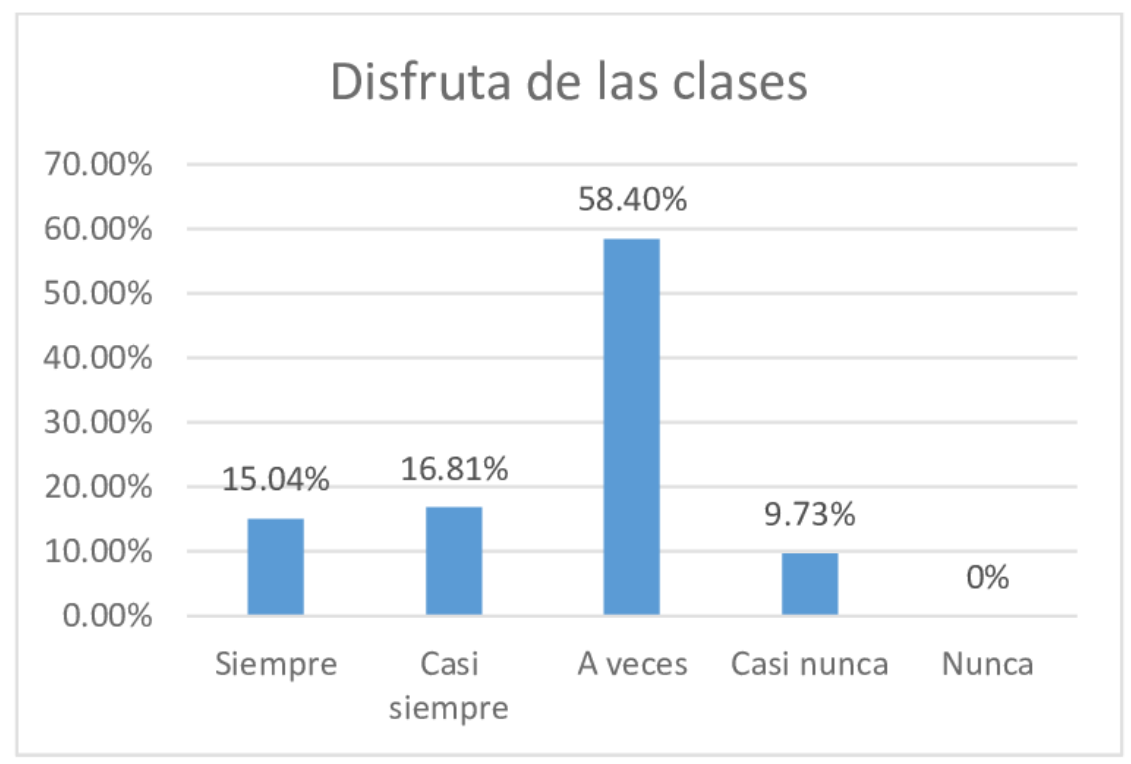

Fuente: Elaboración propia

En cuanto a las herramientas didácticas empleadas con mayor frecuencia por el profesor, se destacan la utilización de maquetas (29.20 \%), seguido del pizarrón (23.00 \%), $\mathrm{y}$ en menor medida las plataformas virtuales y los videos $(12.38 \%$ y $5.30 \%$, respectivamente).

Figura 3. Principales herramientas didácticas empleadas por el profesor

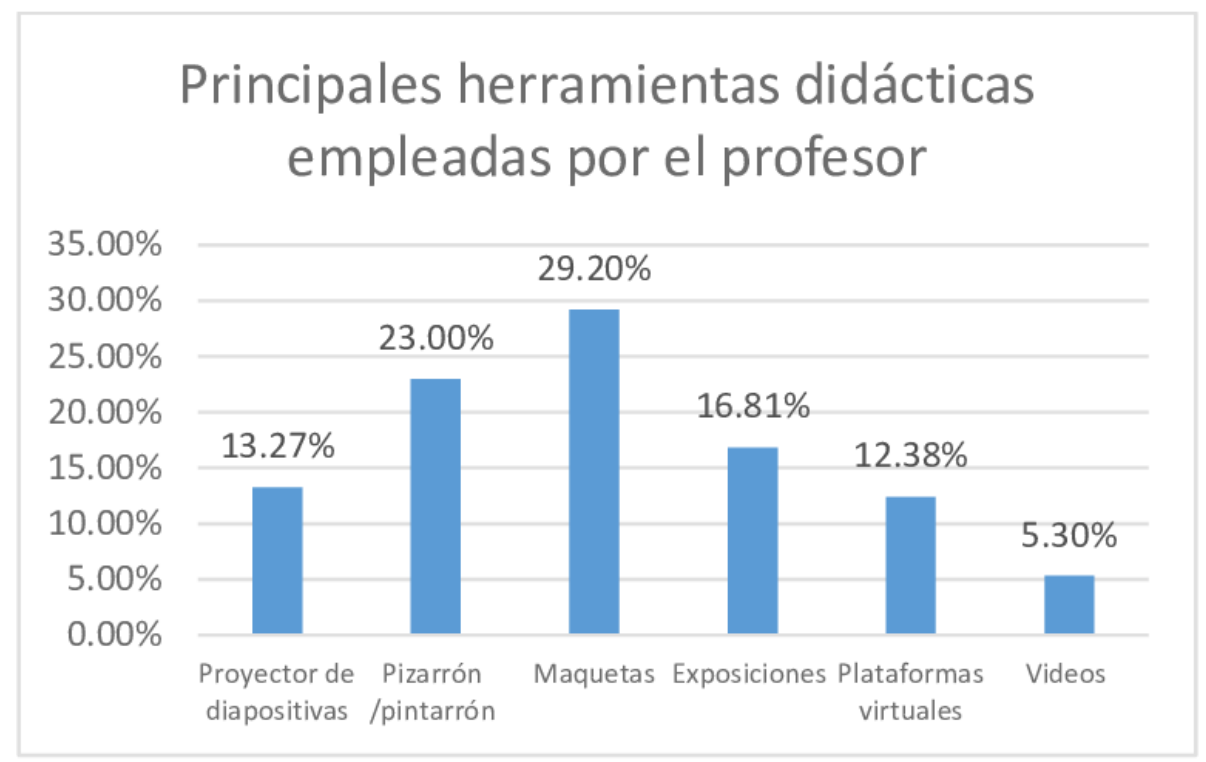

Fuente: Elaboración propia 


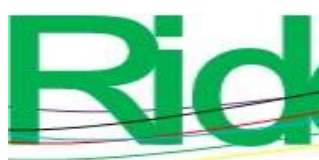

Revista Iberoamericana para la Investigación y el Desarrollo Educativo ISSN 2007 - 7467

Sobre los conocimientos disciplinares, $43.36 \%$ de los encuestados consideró que los profesores no están actualizados, mientras que $37 \%$ comentó que algunas veces, y solo 19.46 \% manifestó que sí lo están.

Figura 4. Percepción del alumno de la actualización del profesor

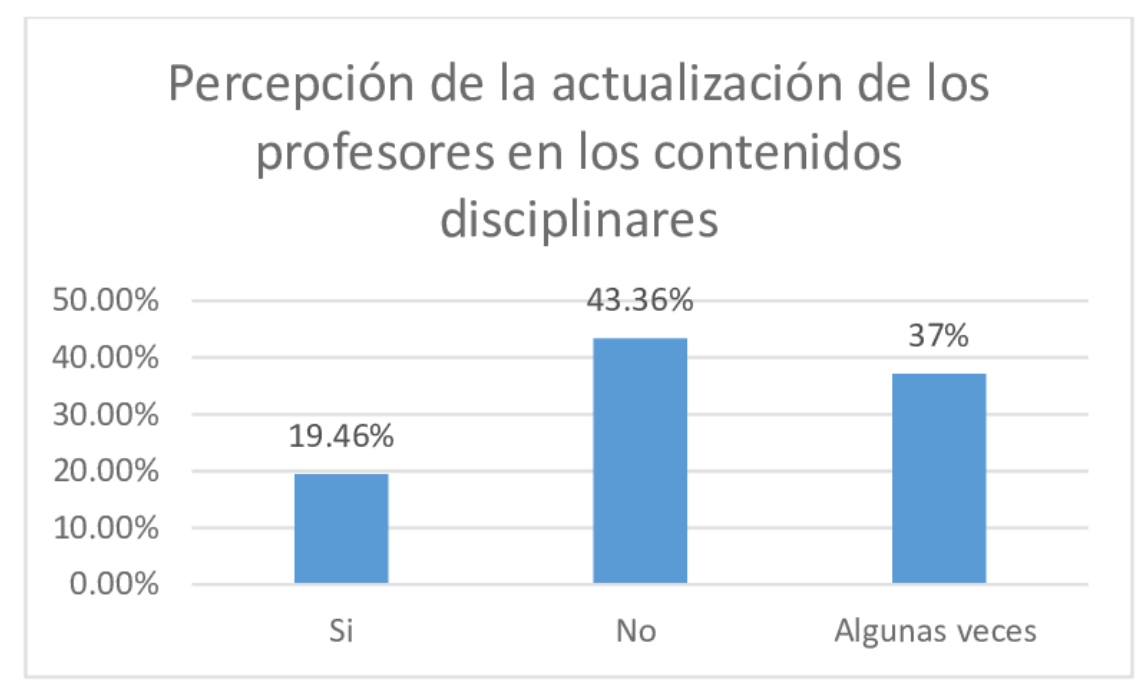

Fuente: Elaboración propia

En lo concerniente a la disponibilidad que tiene el profesor para asesorar al estudiante, $56.63 \%$ opina que no la hay, $25.66 \%$ cree que algunas veces, y solo $17.69 \%$ considera que sí la hay.

Figura 5. Disponibilidad del profesor

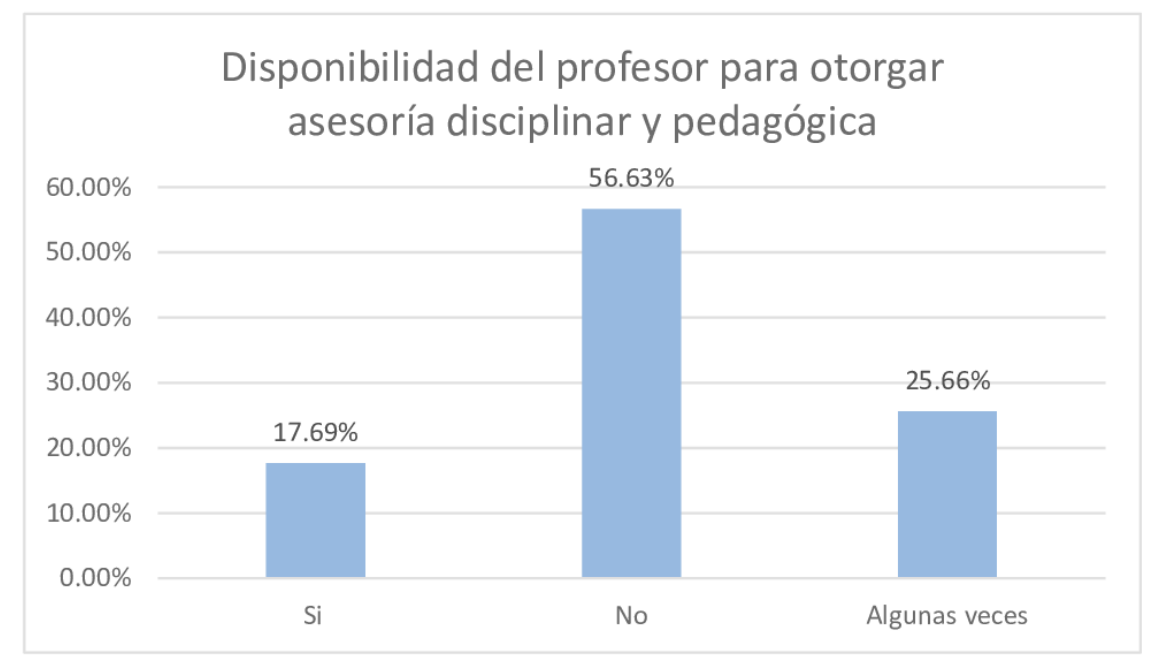

Fuente: Elaboración propia 

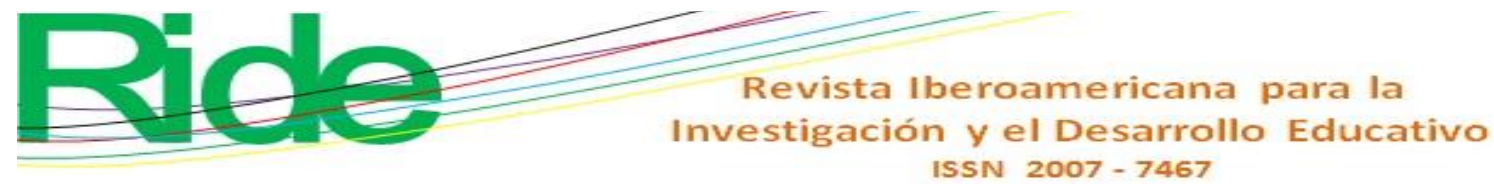

En cuanto a la interacción docente y alumno, se pudieron obtener diversos resultados, ya que al alumno se le permitió seleccionar tres aspectos principales con respecto a los docentes. En tal sentido, $58.0 \%$ considera fundamental la puntualidad, $55.7 \%$ los aspectos relacionados con el conocimiento o saber del profesor, $47.7 \%$ el desarrollo ético, y $36.2 \%$ la tutoría. Por otra parte, se pudo conocer que los participantes no consideraban tan relevantes otros aspectos como la honestidad, la empatía y la motivación.

Figura 6. Percepción de la interacción docente-alumno

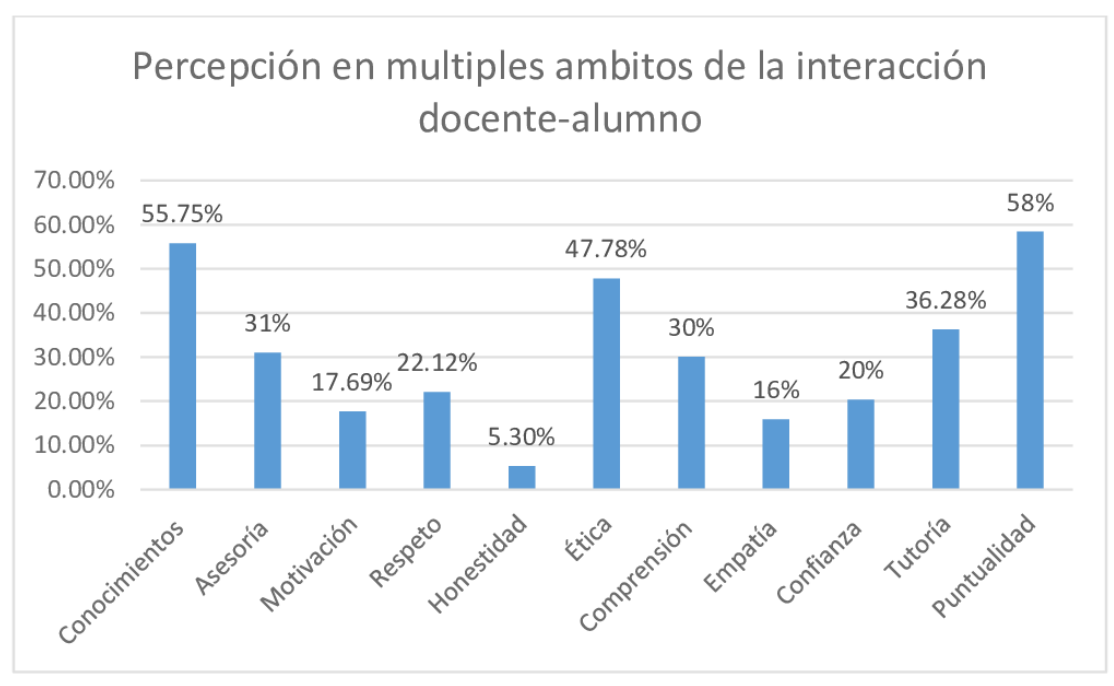

Fuente: Elaboración propia

\section{Discusión}

La simetría de los contenidos temáticos constituye una de las tareas fundamentales dentro del proceso de planeación docente, dado que existen actividades prácticas y teóricas que el alumno debe desarrollar durante su proceso de formación. En este sentido, los contenidos curriculares deben ser impulsores de habilidades y capacidades cognitivas para el desempeño profesional (Alpizar Muni y Molina Naranjo, 2018). Crear los criterios para el alcance de las estrategias curriculares de los contenidos de las asignaturas establece de manera objetiva el avance del aprendizaje en el alumno, por lo que el trabajo metodológico para el desarrollo de la teoría y la práctica debe ser parte del trabajo docente centrado en un nuevo paradigma de planeación didáctica y pedagógica (Vallejo Portuondo, Mendo Alcolea y Lahera Puig, 2019). 


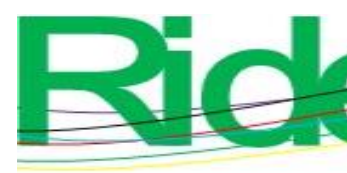

Revista Iberoamericana para la Investigación y el Desarrollo Educativo ISSN 2007 - 7467

En este contexto, gestionar las practicas pedagógicas considerando la integración teoría-práctica de manera equilibrada establecerá la vinculación con el desarrollo de competencias, lo que se traducirá en la inserción productiva del estudiante en su comunidad (Ferreyra, 2018). Los conocimientos teóricos y prácticos son importantes en la apropiación de competencias sólidas y eficaces para la transformación intelectual, social y humana (Damasceno y Ribeiro de Lira, 2018). Sin embargo, como se pudo observar en los resultados encontrados en esta investigación, existe una tendencia hacia el desarrollo de los contenidos práctico por lo cual se considera necesario equilibrar esta percepción en los estudiantes universitarios.

En cuanto al disfrute de las clases, un porcentaje alto de los alumnos encuestados determinó que solo a veces $(58.4 \%$ ) lo hace, lo cual es un factor que debe ser revisado, ya que este aspecto subjetivo es determinante para promover la permanencia de las personas en el aula, así como para asimilar los contenidos. Al respecto, se debe pensar en proponer más variedad de opciones participativas, pues algunos podrían disfrutar más hablando en público, mientras a otros les gustaría más el trabajo en equipo o los retos intelectuales. De hecho, en un estudio de Valerio Ureña y Rodríguez Martínez (2017) se demostró que $29.9 \%$ de los estudiantes disfrutan el desarrollo de la clase cuando el docente fomenta la participación a través de actividades dinámicas, estimulantes y divertidas, variables que hay que considerar en el proceso de formación universitaria. Es cierto que la clase en la enseñanza superior puede tener un carácter teórico o práctico, pero no se debe olvidar que el objetivo principal es impulsar la adquisición de conocimientos y el desarrollo de habilidades y valores, por lo cual deben establecerse escenarios donde el estudiante pueda aprovechar y disfrutar dichos contenidos (Hernández Infante e Infante Miranda, 2017).

Ahora bien, dentro de las principales herramientas didácticas que emplean los profesores se destaca la utilización de maquetas y el pizarrón. En un estudio similar (Teruel, Felipe y Herrera, 2017), se determinó la importancia que tiene el manejo y el uso de las herramientas didácticas, ya que se ha encontrado que $92.6 \%$ de los estudiantes que han usado nuevas herramientas han obtenido una mejor preparación. Esto demuestra la importancia de los materiales didácticos entregados y del ambiente social generado dentro del curso (Teruel et al., 2017). Si bien otros estudios han enseñado que en odontología y medicina los modelos anatómicos y el pizarrón ofrecen buenos resultados (Morales García, Priego Álvarez, Ávalos García y Córdova Hernández, 2014), no se deben descuidar otras opciones más actuales para el reforzamiento del aprendizaje. 


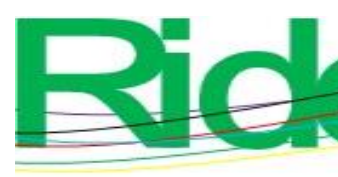

Revista Iberoamericana para la Investigación y el Desarrollo Educativo ISSN 2007-7467

Por otra parte, la percepción de los alumnos en relación con la actualización de los profesores sobre los contenidos disciplinares resultó ser negativa. Cabe destacar que esta apreciación pudiera deberse a que es muy común que los alumnos exijan mayor conocimiento de los docentes incluso en áreas cognitivas que aún no corresponden a su proceso de su formación (Valerio Ureña et al., 2017). Aun así, este es un factor que se debe atender, puesto que un profesor con un perfil innovador influye en la motivación del alumno (Díaz Yáñez y Sánchez, 2017).

En relación con la asesoría disciplinar y pedagógica otorgada por el profesor, los alumnos expresaron que hay una baja disponibilidad. Esto también se debe atender porque en la actualidad el acompañamiento formativo mediante la asesoría y la tutoría resultan vitales para conseguir la formación integral del estudiante. Para lograr esto, sin embargo, el profesor debe reconocer al estudiante como un individuo complejo que se halla inmerso en un contexto cambiante (Klug y Peralta, 2019).

En el ámbito de la relación docente-alumno existen factores que se consideran importantes, ya que es lo que espera el alumno del profesor. En este estudio se destacan como los aspectos más sobresalientes la puntualidad, el conocimiento, la ética y las tutorías. Al respecto, se ha determinado que los valores individuales y profesionales se fortalecen en el ámbito formativo, lo cual requiere de un conjunto de acciones por parte del profesor. De lo contrario, si carece o no tiene claro sus valores personales, puede ser deficiente en la transferencia de valores exigidos en el proceso de formación profesional (Expósito, 2018).

En síntesis, se puede afirmar que este estudio solo ofrece una aportación mínima sobre la importancia de conocer las necesidades y expectativas del estudiante de educación superior, por lo que puede ser concebido como una indagación diagnóstica para dibujar las competencias y habilidades requeridas por el profesor universitario.

En cuanto a las limitaciones de este trabajo, se podría señalar el abordaje metodológico, el cual se podría complementar con enfoques cualitativos. Finalmente, la principal debilidad se encontraría en la escasa participación de los responsables de la planeación y ejecución de los programas de estudios. 


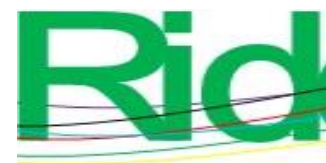

Revista Iberoamericana para la Investigación y el Desarrollo Educativo ISSN 2007-7467

\section{Conclusiones}

Los cambios en las teorías del aprendizaje y los nuevos paradigmas en la construcción de saberes para el desarrollo de futuros profesionales en las instituciones de educación superior — en congruencia con la dinámica social, política y demográfica— han gestado una nueva concepción de la práctica docente al reconocer un empoderamiento del estudiante, el cual ahora puede tener una mejor relación con su profesor, proponer los contenidos curriculares, las actividades extracurriculares y conocer a las instituciones educativas que mejor se ajustan a su proceso de formación. Esto, lógicamente, ha obligado a las instituciones de educación superior a ser más competitivas y a mejorar sus programas de estudios, así como la planta docente, la infraestructura y los mecanismos de evaluación. Por ello, resulta esencial establecer mecanismos de capacitación constantes para los docentes, así como iniciativas innovadoras que se ajusten a las necesidades de los alumnos. Este conocimiento continuo de estas expectativas contribuirá a la formación de mejores profesionales capaces de insertarse con efectividad y eficacia en el contexto laboral. 


\section{Referencias}

Alpizar Muni, J. y Molina Naranjo, M. (2018). Las competencias en el contexto de la Educación Superior del Ecuador. Atenas, 2(42), 108-121. Recuperado de https://www.redalyc.org/articulo.oa?id=4780/478055152008

Arce-Medina, E. (2008). Táctica centrada en los alumnos mejora el aprendizaje. Innovación Educativa, $\quad$ 8(43), 25-31. Recuperado de https://www.redalyc.org/articulo.oa?id=1794/179421221003

Blancas Torres, E. (2018). Educación y desarrollo social. Horizonte de la Ciencia, 8(14), 113-121. Recuperado de https://www.redalyc.org/articulo.oa?id=5709/570960866008

Damasceno, E. y Ribeiro de Lira, M. (2018). Formación de profesores, plan de estudios y práctica pedagógica: enseñanza interdisciplinaria. Educere, 22(72), 283-293. Recuperado de https://www.redalyc.org/articulo.oa?id=356/35656041002

Díaz Yáñez, M. y Sánchez, G. (2017). El trabajo docente desde las percepciones de profesores en formación. Educere, 21(69), 427-437. Recuperado de https://www.redalyc.org/articulo.oa?id=356/35655222015

Expósito, C. (2018). Valores básicos del profesorado: una aproximación desde el modelo axiológico de Shalom Schwartz. Educación y Educadores, 21(2), 307-325. Recuperado de https://www.redalyc.org/articulo.oa?id=83460719007

Ferreyra, H. (2018). Escuela secundaria: currículo, saberes y prácticas en contexto. Sophia, 14(1), 1-11. Recuperado de https://www.redalyc.org/articulo.oa?id=4137/413755833008

García-Rangel, E., García Rangel, A. y Reyes Angulo, J. (2014). Relación maestro alumno y sus implicaciones en el aprendizaje. Ra Ximhai, 10(5), 279-290. Recuperado de https://www.redalyc.org/articulo.oa?id=461/46132134019

Guerra Santana, M., Rodríguez Pulido, J. y Rodríguez, J. (2019). Aprendizaje colaborativo: experiencia innovadora en el alumnado universitario. REXE, Revista de Estudios y Experiencias en Educación, 18(36), 269-281. Recuperado de https://www.redalyc.org/articulo.oa?id=2431/243158860016

Hernández Infante, R. e Infante Miranda, M. (2017). La clase en la educación superior, forma organizativa esencial en el proceso de enseñanza-aprendizaje. Educación y 


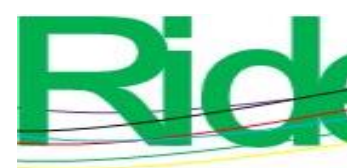

Revista Iberoamericana para la Investigación y el Desarrollo Educativo ISSN 2007-7467

Educadores, 20(1), 27-40. Recuperado de https://www.redalyc.org/articulo.oa?id=834/83449754002

Klug, M. y Peralta, N. (2019). Tutorías universitarias. Percepciones de estudiantes y personal tutor sobre su uso y funcionamiento. Revista Electrónica Educare, 23(1), 1-23. Recuperado de https://www.redalyc.org/articulo.oa?id=1941/194159163016

Liesa, Eva, \& Castelló, Monserrat, \& Becerril, Lorena (2018). Nueva escuela, ¿nuevos aprendizajes?. REXE. Revista de Estudios y Experiencias en Educación, 2(1),1529.[fecha de Consulta 2 de Junio de 2020]. ISSN: 0717-6945. Disponible en: https://www.redalyc.org/articulo.oa?id=2431/243154900001

Molina, M. (2018). Prácticas de enseñanza que entraman escritura con contenidos: perspectivas de alumnos universitarios de dos disciplinas. Propuesta Educativa, 1(49), 61-72. Recuperado de https://www.redalyc.org/articulo.oa?id=4030/403060198007

Morales García, M., Priego Álvarez, H., Ávalos García, I. y Córdova Hernández, J. (2014). Acción docente: una perspectiva del alumno. Perspectivas Docentes, O(49). Doi: https://doi.org/10.19136/pd.a0n49.580

Moreno Olivos, T. (2018). La evaluación docente en la universidad: visiones de los alumnos. REICE. Revista Iberoamericana sobre Calidad, Eficacia y Cambio en Educación, 16(3), 87-102. Recuperado de https://www.redalyc.org/articulo.oa?id=551/55160077005

Teruel, M., Felipe, A. y Herrera, M. (2017). Estrategias didácticas para mejorar la calidad de la enseñanza y el aprendizaje de los estudiantes del Curso Histología, Embriología y Teratología. Espacios en Blanco. Revista de Educación, (27), 153-176. Recuperado de https://www.redalyc.org/articulo.oa?id=3845/384551991007

Valerio Ureña, G. y Rodríguez Martínez, M. (2017). Perfil del profesor universitario desde la perspectiva del estudiante. Innovación Educativa, 17(74), 109-124. Recuperado de https://www.redalyc.org/articulo.oa?id=1794/179452787007

Vallejo Portuondo, G., Mendo Alcolea, N. y Lahera Puig, M. (2019). Procedimientos didáctico-metodológicos para la implementación de las estrategias curriculares en la educación superior. MEDISAN, 23(2), 360-371. Recuperado de https://www.redalyc.org/articulo.oa?id=3684/368459444014

Zabalza Beraza, M. A. (2011). Metodología docente. Revista de Docencia Universitaria. REDU, 9(3), 75-98. Recuperado de http://redaberta.usc.es/redu 


\begin{tabular}{|c|c|}
\hline Rol de Contribución & Autor (es) \\
\hline Conceptualización & $\begin{array}{l}\text { Manuel Higinio Morales García (principal), Carlos Alberto } \\
\text { Balcázar Nájera (apoyo) }\end{array}$ \\
\hline Metodología & Manuel Higinio Morales García (principal) \\
\hline Software & NO APLICA \\
\hline Validación & $\begin{array}{l}\text { Manuel Higinio Morales García (principal), Heberto Romeo } \\
\text { Priego Álvarez (principal), }\end{array}$ \\
\hline Análisis Formal & $\begin{array}{l}\text { Carlos Alberto Balcázar Nájera (principal), Jonatan Flores } \\
\text { Morales (apoyo), Manuel Higinio Morales García (principal). }\end{array}$ \\
\hline Investigación & $\begin{array}{l}\text { Carlos Alberto Balcázar Nájera (principal), Jonatan Flores } \\
\text { Morales (apoyo), Manuel Higinio Morales García (principal), } \\
\text { Heberto Romeo Priego Álvarez (apoyo) }\end{array}$ \\
\hline Recursos & NO APLICA \\
\hline Curación de datos & $\begin{array}{l}\text { Manuel Higinio Morales García (apoyo), Heberto Romeo } \\
\text { Priego Álvarez (principal). }\end{array}$ \\
\hline $\begin{array}{l}\text { Escritura - Preparación del } \\
\text { borrador original }\end{array}$ & $\begin{array}{l}\text { Manuel Higinio Morales García (principal), Heberto Romeo } \\
\text { Priego Álvarez (principal). }\end{array}$ \\
\hline $\begin{array}{l}\text { Escritura - Revisión y } \\
\text { edición }\end{array}$ & $\begin{array}{l}\text { Manuel Higinio Morales García (principal), Heberto Romeo } \\
\text { Priego Álvarez (principal) }\end{array}$ \\
\hline Visualización & $\begin{array}{l}\text { Carlos Alberto Balcázar Nájera (principal), Jonatan Flores } \\
\text { Morales (apoyo), Manuel Higinio Morales García (principal), } \\
\text { Heberto Romeo Priego Álvarez (principal) }\end{array}$ \\
\hline Supervisión & $\begin{array}{l}\text { Manuel Higinio Morales García (principal), Heberto Romeo } \\
\text { Priego Álvarez (apoyo). }\end{array}$ \\
\hline $\begin{array}{l}\text { Administración de } \\
\text { Proyectos }\end{array}$ & Manuel Higinio Morales García \\
\hline Adquisición de fondos & NO APLICA \\
\hline
\end{tabular}

